\title{
Germanica
}

\section{Le naufrage existentiel d'un vieil homme dans une société technique et industrielle développée : Arne Ross :Pauls Fall (2006)}

An Old Man's Existential Wreck in a Technical and Industrial Developed Society

Die genheiterte Existenz eines alten Mannes in einer hoch entwickelten

technischen und industriellen gesellschaft

\section{Bernard Bach}

\section{OpenEdition}

Journals

Édition électronique

URL : http://journals.openedition.org/germanica/1579

DOI : 10.4000/germanica. 1579

ISSN : 2107-0784

\section{Éditeur}

Université de Lille

\section{Édition imprimée}

Date de publication : 10 juillet 2012

Pagination : 109-121

ISBN : 9782913857292

ISSN : 0984-2632

\section{Référence électronique}

Bernard Bach, « Le naufrage existentiel d'un vieil homme dans une société technique et industrielle développée : Arne Ross :Pauls Fall (2006) », Germanica [En ligne], 50 | 2012, mis en ligne le 19 juillet 2012, consulté le 06 octobre 2020. URL : http://journals.openedition.org/germanica/1579 ; DOI : https://doi.org/10.4000/germanica.1579

Ce document a été généré automatiquement le 6 octobre 2020.

(c) Tous droits réservés 


\title{
Le naufrage existentiel d'un vieil homme dans une société technique et industrielle développée : Arne Ross :Pauls Fall (2006)
}

\author{
An Old Man's Existential Wreck in a Technical and Industrial Developed Society \\ Die genheiterte Existenz eines alten Mannes in einer hoch entwickelten \\ technischen und industriellen gesellschaft
}

\section{Bernard Bach}

L'histoire de la vieillesse donne à voir de cet âge de la vie une prédominance de stéréotypes à connotation négative ${ }^{1}$. La vieillesse est ainsi le plus souvent associée à la déchéance physique, intellectuelle, à la maladie, à la solitude, à la dépendance. Dans le monde occidental industrialisé et urbanisé, l'accélération du vieillissement démographique suscite à la fois des craintes liées au financement des retraites ou à la prise en charge du très grand âge et des appels à changer radicalement le regard sur la vieillesse, à en finir avec une vision pessimiste et erronée de la vieillesse, à inventer un nouvel art de vivre ensemble ${ }^{2}$.

La question de la vieillesse est peut-être plus cruciale en Allemagne qu'ailleurs en raison d'un déclin démographique prononcé et d'un vieillissement rapide de la population. En témoignent, entre autres, les multiples ouvrages de toutes natures sur la vieillesse qui paraissent dans les rayons des librairies allemandes. Dans la production littéraire du xxie siècle, le thème de la vieillesse est, lui aussi, très présent. Même une exploration rapide laisse entrevoir que l'on est assez loin de la représentation d'un bonheur assumé et affiché de la vieillesse. Dans la production littéraire allemande des dix dernières années, la vieillesse est souvent traitée sous l'angle de la déchéance, de la maladie, de la souffrance, de la dépendance ${ }^{3}$.

Le récit Pauls Fall d'Arne Ross", outre sa grande qualité d'écriture ${ }^{5}$, se distingue par l'attention portée à la dimension existentielle de la vieillesse plus qu'à la déchéance 
physique, même si celle-ci est présente et que le récit se termine sur la mort du personnage principal. Cette dimension existentielle ne peut pas ne pas être en relation et en interaction avec la société dans laquelle évolue le sujet. En somme la représentation de la vieillesse qui se dégage de ce récit est révélatrice du mode de fonctionnement du système dans lequel vit Paul. Le récit d'Arne Ross donne à voir l'ultime journée (un 20 octobre) de la vie d'un vieil homme habitant dans un lotissement bien propre et menant l'existence de Monsieur Tout-le-monde. L'indétermination relative de son identité en fait une figure paradigmatique du «vieillir » dans la société allemande postmoderne. Cette figure pourrait bien dénoncer l'échec de cette société allemande qui, sur la base de sa "performance économique globale ", peut s'enorgueillir d'être la " troisième économie nationale du monde " ${ }^{6}$ et se flatter d'avoir «la meilleure image mondiale » ${ }^{7}$. Le titre du livre Pauls Fall indique que la vieillesse de cet homme est un naufrage et qu'il s'achemine inéluctablement vers sa mort. La vie de Paul est marquée par le poids d'un passé jamais clairement explicité, difficilement assumé, son existence présente apparaît vide de sens et dépourvue d'intériorité et son avenir barré se présente comme la chronique d'une mort annoncée.

\section{Le poids d'un passé jamais explicité}

Le personnage central du récit est un vieil homme dont le lecteur n'apprend que le prénom Paul. Rien n'est dit explicitement sur l'histoire de cet homme, sa situation sociale, sa carrière professionnelle, ses aspirations, ses angoisses. Une identité incertaine se dessine au fil de ce que l'on apprend sur sa vie sentimentale, les relations superficielles et les contacts furtifs qu'entretient Paul avec un certain nombre de personnes qui appartiennent toutes au même monde des seniors que lui. Le monde de Paul, un monde en retrait, est celui d'un certain type de seniors liés entre eux par un passé qu'ils ont tendance à ressasser, idéaliser, regretter, et souffrant des mêmes maux de la vieillesse. Un passé lourd, jamais explicitement nommé, semble peser sur le vieil homme et affecter son comportement présent. Ce passé affleure au travers de sa vie sentimentale présente, de son réseau relationnel restreint et au travers de ses réactions face aux manœuvres militaires qui ont lieu dans son environnement immédiat.

$\mathrm{Au}$ début du récit, le lecteur découvre le personnage Paul en train de lire le journal dans sa cuisine (PF 7-8), exactement comme son voisin d'en face (PF 12), ainsi s'esquisse un mode de vie des seniors. Celle qui semble être son épouse et revient des courses ne porte pas de nom, elle est désignée par l'initiale G. (PF 15). Dans la vie de ce vieil homme, elle ne semble plus avoir de véritable existence, elle apparaît comme une sorte de fantôme d'un bonheur passé. Très rapidement se dessine le stéréotype du couple traditionnel : lui lit le journal pendant qu'elle range les courses (PF 15), lave la vaisselle (PF 17). Ce qui frappe dans ce couple, c'est l'indifférence de Paul, il ne regarde pas son épouse $^{8}$, ne l'écoute pas, ne lui répond pas, ne l'aide pas (PF 15, 16, 17), lui reproche de s'être attardée (PF 16) et manifeste sa mauvaise humeur quand elle lui demande de porter un gâteau au professeur Schneider (PF 18). D'emblée se donne à voir le profil d'un vieillard d'humeur grincheuse, tout le contraire de la joie de vivre. Les quelques mots qu'échange le couple laissent entrevoir une relation tendue, sans tendresse. Bref, le lecteur a le sentiment d'être en présence d'un vieux couple sans illusions dont l'amour a cédé la place à de sourdes rancœurs et aux seules préoccupations matérielles. Cette situation de tension trouve un élément d'explication dans la suite du récit. Paul 
rend en effet visite à une femme prénommée Ingeborg, manifestement une maîtresse avec qui il entretient une relation ancienne, ce que n'ignore pas G. (PF 164). Le fait que son prénom soit mentionné pourrait être l'indice d'une relation plus positive, ou du moins d'un niveau de reconnaissance plus élevé de cette femme.

Mais l'état de la relation entre Paul et Ingeborg semble aussi délabré que celui entre Paul et G. La visite de Paul contrarie manifestement Ingeborg (PF 126-127). Il apparaît assez rapidement que cette relation est, elle aussi, tendue. En dépit de tentatives répétées, la conversation s'enlise (PF 135), un silence lourd de sous-entendus (PF 138, 139, 140, 141) s'établit entre eux, la rancœur semble s'être installée également au cœur de ce couple (PF 136, 147). L'attitude de Paul est faite de froideur (PF 126, 138), d'indifférence (PF 127), elle est empreinte de cynisme (PF 127, 128), il jette sur Ingeborg un regard sans complaisance (PF 126, 139). Au moment où Ingeborg s'étouffe avec une arrête de poisson, Paul détourne pudiquement le regard (PF 137), il reste indifférent au geste de tendresse qu'elle esquisse un peu plus tard (PF 139). Paul évoque son fils avec insistance (PF 138-139), mais Ingeborg ne se sent manifestement pas concernée (PF 139, 140). À vrai dire, quand Ingeborg s'adresse à Paul, elle le fait systématiquement sur le mode de l'injonction (PF 128, 130, 132, 133), traduisant ainsi une relation de domination ou une forme d'instrumentalisation de la relation. Après avoir dîné ensemble, Paul s'endort sur le canapé (PF 142, 143, 144, 145) tandis qu'Ingeborg s'enivre de vin (PF 142) et finit par casser la vaisselle (PF 144). Cette relation sans tendresse semble fonctionner sur le mode du ressentiment, de l'injonction, de la domination, voire de la provocation.

La vie sentimentale de Paul semble être un échec, elle donne à voir en quelque sorte une réification de la relation, il continue à entretenir la relation avec ces deux femmes sans doute davantage par habitude, par commodité que par choix. Elles font en quelque sorte partie de sa vie, même si leur dimension vivante appartient au passé. Elles semblent peser sur sa vie quotidienne. Paul lui-même n'exprime aucun avis verbal sur ces deux relations, il ne les remet pas en question, elles font tout simplement partie de sa vie. Mais il est d'autant plus frappant de constater à quel point l'attitude de Paul exprime le désir d'échapper à l'enfermement de ces relations. En présence de G. comme d'Ingeborg, son regard se détourne constamment des femmes et s'échappe vers l'extérieur (PF 15, 16, 18, 129, 134, 138, 141, 146), expression muette de son malaise et du désir de se libérer de ces relations pesantes et aliénantes. Son âge et son état physique ne l'autorisent sans doute pas à espérer construire une nouvelle relation plus épanouissante. Sentimentalement Paul ne semble plus avoir d'avenir, il semble résigné à supporter dans le présent ce qui appartient au passé.

Paul évoque à plusieurs reprises un fils qui semble résider à Berlin et dont les relations se limitent à des coups de téléphone épisodiques (PF 24,173) et à un échange épistolaire assez régulier (PF 138). En somme, la famille semble s'être disloquée, elle aussi appartient au passé, le lien intergénérationnel semble rompu. Paul n'exprime aucun regret, il accepte cette situation comme un fait.

Un autre passé affleure par ailleurs au cours de la visite que rend Paul au professeur Schneider, un homme âgé, comme lui. Le professeur Schneider s'efforce d'entamer une conversation avec Paul, qui ne cesse de s'évader dans les rêveries et n'entend que des bribes de phrases ou de souvenirs qui se perdent dans le silence. À vrai dire, le professeur n'attend pas de réponse de Paul, il poursuit une conversation avec luimême, son monde, ses fantômes. Les fragments d'une mémoire éclatée qu'enregistre Paul laissent tout au plus deviner une sombre vision du présent (PF 72, 156), ainsi que 
des regrets concernant la disparition d'un ordre stable et rassurant (76) et une sorte de nostalgie de l'après-guerre (PF 77). Le professeur Schneider dévoile en outre un aspect peu reluisant de son passé, celui de voleur d'objets dans les magasins (PF 81), et la manière dont un cul-de-jatte l'a remis dans le droit chemin (PF 81-82). Le fait que Paul dérobe un briquet en or (PF 89) au professeur durant la visite l'apparente étrangement à ce passé de délinquant. Lorsqu'il montrera le briquet à G. (PF 172), il se verra opposer une désapprobation muette à laquelle il ne réagit que mollement, mais qui pourrait donner à penser que ce penchant pour le vol est ancien et connu de G.

$\mathrm{Au}$ cours de son périple, Paul rencontre un homme ivre qui, l'ayant vu se débattre contre des insectes, pense qu'il est dans le même état que lui (PF 154), ce qui suscite en lui une arrière-pensée d'entente. Mais il s'établit très rapidement entre les deux hommes un rapport de domination, l'homme hurle des ordres, se fait menaçant (PF 155, 156). Paul croit reconnaître cet homme (PF 158), qui fait le salut militaire et lui rappelle en somme le passé problématique de l'Allemagne. L'homme devient franchement inquiétant lorsqu'il revient vers Paul avec un ricanement sardonique (PF 160) et se transforme en une sorte d'homme-chien emporté par une violence extrême et incontrôlable (PF 160) qui s'achève par un long hurlement jusqu'à l'extinction de sa voix (PF 161). Paul assiste à la scène sans faire aucun commentaire, il presse simplement un peu le pas pour rentrer chez lui (PF 161). En fait Paul participe d'une certaine manière de ce monde évoqué par le comportement de l'homme ivre. À deux autres endroits du récit, il fait, lui aussi, le salut militaire: quand le professeur Schneider évoque devant lui la "crainte de Dieu comme premier pas vers la sagesse " (PF 86), Paul répond étrangement par un salut militaire induisant une forme de sacralisation du chef militaire. Au cours de sa promenade, Paul monte sur une colline et contemple le paysage qui s'étend sous ses yeux, cette position l'incite à se mettre alors au garde-à-vous (PF 102) avec la nette impression de s'exposer devant quelqu'un. Cette expression d'un rapport de subordination sans raison apparente donne à penser que le mode de comportement militaire intériorisé dans son passé continue d'être actif en Paul. À cela s'ajoute la brutalité avec laquelle Paul traite le chien malade du professeur Schneider (PF 67). Le lecteur n'apprend rien sur le passé de Paul, mais la non-réaction de Paul vis-à-vis de l'homme ivre (une forme de complicité ?) ainsi que son propre comportement laissent entrevoir un aspect inquiétant de cette personnalité, qui semble renvoyer non seulement à son histoire personnelle mais aussi à l'histoire de son pays. Le vieil homme apparaît tributaire d'un contexte historique et politique qui pour être tabou n'en reste pas moins actif en lui. Il ne semble pas avoir fait, au niveau individuel, le nécessaire travail critique sur son implication dans l'histoire allemande que le lecteur peut supposer sur la base de son comportement.

Cet arrière-plan historique est également présent à travers la réaction de Paul face aux manœuvres militaires qui ont lieu dans son environnement immédiat. Tout au long du récit Paul enregistre ce qu'il appelle « les bruits de guerre »(PF 28, 150, 27, 39, 97), il suit notamment de manière attentive l'évolution des hélicoptères (PF 97). À un moment donné, Paul vit les simulations d'attaque des avions comme une scène de guerre réelle, il se sent anéanti par le bruit infernal, il enfonce sa tête dans les épaules, se recroqueville sur lui-même comme s'il se terrait dans un creux (PF 116), il est blême, pris de panique, trempé de sueur (PF 117). Le lecteur comprend que le passage des avions a réveillé en Paul des fantômes anciens. Au moment où Paul note que les « bruits de guerre » se sont tus, il a recours au vocabulaire militaire pour décrire sa lutte contre les insectes (PF 151). C'est comme s'il ne parvenait pas à se libérer de ce passé de guerre 
et que les réflexes de guerre étaient toujours présents en lui. Cela explique aussi son inquiétude face aux bruits et cris de toutes sortes (PF 32, 35, 45, 66), c'est comme si la menace d'une attaque planait en permanence dans sa tête. Paul continue de vivre dans un passé tabou. En somme, ce vieil homme apparaît en grande partie agi par son passé. Il n'y a chez lui aucun retour sur son passé, aucune prise de distance critique, aucun regret. Il s'est résigné à accepter la triste existence qui résulte pour lui de ce passé. Sa vie semble vide de sens, sans révolte, sans aspiration, sans projets, sans joie ${ }^{9}$.

\section{Une existence présente vide de sens et dépourvue d'intériorité}

Tout au long du récit, le narrateur donne des indications sur la déchéance physique de Paul : mains ridées (PF 13), tremblements (PF 10,14), souffle court (PF 19, 57, 90, 124), claudication intermittente (PF 14,70), difficultés à se mouvoir (PF 124), à s'asseoir et à se relever d'un fauteuil (PF 70), maladresses ${ }^{10}$, manque d'assurance (PF 32), douleurs (PF 92, 181). Le narrateur note une certaine négligence de soi chez Paul (PF 21), signe d'une image dégradée de lui-même. Mais ce qui caractérise le plus fortement ce vieil homme, c'est la lenteur (PF 7, 13, 20, 21-22, 61) qui imprime son rythme à tout le récit ${ }^{11}$. Paul vit au ralenti, il met un temps interminable à réaliser les gestes quotidiens, à prendre une décision, à se mettre en route. G. le lui fait remarquer sans aménité (PF 23).

$\mathrm{Au}$ début du récit, Paul est installé dans la cuisine devant son journal, il lit, relit, souligne en bleu, en rouge (PF 9, 10), ne cesse de scruter la pendule, de regarder par la fenêtre, de soupirer, de reprendre sa lecture. Bref, il se dégage d'emblée une atmosphère d'ennui, de lassitude, de vide. L'activité de lecture semble vide de sens, comme d'ailleurs l'ensemble des activités de sa dernière journée de vie. Il semble s'ennuyer de lui-même, devenu incapable de s'inscrire dans une temporalité finalisée. Tout indique qu'au soir de sa vie, cet homme, en perpétuelle fuite devant lui-même (PF 52), est confronté à l'absence de signification, il semble devenu une " temporalité vide et insensée $»^{12}$. Tout au long du récit Paul, comme étranger au monde, ne cesse de promener un regard circulaire interrogateur sur ce monde muet (PF 8, 14, 54, 69, 77, 130), de scruter un ciel changeant qui ne suscite aucune émotion en lui (PF 76, 90, 100, $119,156)$. Quand son regard se dirige vers le haut, il est aveuglé par la clarté du soleil (PF 60, 69), quand il se dirige vers le bas il ne rencontre que les ténèbres (PF 132, 133). Avec une sorte d'indifférence absolue, il enregistre tous les bruits d'un monde technique qui n'est finalement qu'agression lancinante ${ }^{13}$, dissonances stridentes (PF 116), tonitruances effrayantes (PF 116). Paul ne cesse de constater la vacuité des lieux qu'il traverse, l'absence de vie humaine, de mouvement autour de lui, son environnement semble le renvoyer en permanence à sa situation de solitude (PF 36, 57, $102,59,60,77)$, de retrait par rapport au monde des actifs. Ses tentatives pour entrer en communication avec des personnes sont vouées systématiquement à l'échec (PF 78-79). Quand il salue quelqu'un il n'obtient pas de réponse (PF 41, 55, 56, 61, 89, 96), quand il pose une question, il se heurte au mutisme, quand il croit avoir établi un contact avec des personnes, il s'avère qu'on ne le regarde même pas et qu'on se détourne de lui ( $\mathrm{PF}$ 61,99). Quand le téléphone sonne, il s'étonne tant il a pris l'habitude d'un téléphone silencieux (PF 24). Paul est confronté à l'indifférence du monde et des hommes, il est ainsi vidé de son humanité. Au cours de sa dernière journée de vie, il rencontre ou 
croise près d'une vingtaine de personnes, pour la plupart âgées comme lui, il ne réussit à engager une conversation avec aucune d'entre elles.

Dans cette temporalité vide de sens et de relations, Paul est taraudé par une angoisse inexprimée qui se traduit dans le rythme saccadé de sa marche: Paul traîne le pas, accélère, court, s'arrête, hésite, repart sans raisons apparentes comme s'il était agi par une force intérieure ( $\mathrm{PF} 42,52,57,90,107,121$ ), mais cette marche hésitante, désordonnée, sans but conduit invariablement à des impasses obligeant Paul à revenir sur ses pas (PF 59, 91). Pour calmer l'angoisse, il reste le « plaisir » de manger : Paul est saisi d'une véritable crise boulimique et il avale en entier le gâteau qu'il était chargé d'apporter au professeur Schneider (PF 43, 45, 47, 48).

Paul évolue dans un univers clos sur lui-même et oppressant, il y enregistre par intermittence les accessoires ou habitudes qui signifient la transcendance : la nouvelle chapelle ( $\mathrm{PF} 58,93$ ), une affiche avec l'indication des offices religieux ( $\mathrm{PF}$ 62), la prière avant le repas (PF 71, 165-166), les cloches (PF 94), le chant d'un choral (PF 153). Paul note en somme la présence d'un patrimoine religieux, mais qui est devenu sans signification pour lui ${ }^{14}$. La brève rencontre avec le pasteur dans le cimetière souligne l'abîme entre le monde du pasteur et celui de Paul (PF 95). Dans l'univers de Paul la religion a perdu sa capacité à être pourvoyeuse de sens, le vieil homme est marqué par le « désenchantement du monde » (Max Weber).

Si Paul n'exprime aucun état d'âme, aucune réflexion critique sur sa situation ni celle de la société, aucune velléité de changement, toute son attitude indique cependant qu'il ne parvient pas à acquiescer à ce présent vide de sens. Paul ne cesse d'enregistrer les signes d'enfermement ${ }^{15}$, le regard circulaire déjà évoqué, qui se referme régulièrement sur l'observateur (PF 38, 47, 69, 106, 176), apparaît comme l'expression muette et inquiète du désir d'échapper à cet univers absurde ( $\mathrm{PF} 8,52,54,77,80)$. Le plus souvent ce regard s'échappe par une fenêtre, une porte, en quête d'un impossible ailleurs, s'arrêtant sur les jardins comme s'il s'agissait de retrouver l'Éden perdu, puis s'égarant dans le vide (PF 16, 54, 77, 80, 84, 129, 134, 138, 176). Sans raison apparente, Paul est poussé à pénétrer dans les jardins ( $\mathrm{PF} 62$ ).

S'il n'y a pas chez Paul de retour sur soi, de questionnement existentiel, d'aspiration à changer la vie, bref si Paul semble se réduire à l'extériorité multiple et dispersée du monde, sa scrutation obsessionnelle de l'intérieur des maisons ( $\mathrm{PF} 39,40)$ indique cependant un mouvement vers l'intériorité. Si l'on admet avec Bachelard que la maison signifie l'être intérieur ${ }^{16}$, on peut comprendre l'obsession de Paul comme une tentative désespérée de réveiller l'homme intérieur et de se libérer de la réification du moi. Mais la tentative est vaine, Paul ne voit que des intérieurs éclairés, mais vides, qui ramènent son regard vers l'extériorité bruyante ${ }^{17}$. À un autre moment, Paul scrute un intérieur plongé dans les ténèbres, il sonne, insiste (PF 120-121) avant de prendre littéralement la fuite comme s'il avait commis un délit. Au cours de sa promenade, il pénètre dans une clairière, autre symbole de l'intériorité, au centre de laquelle il découvre, outre des déchets de la civilisation industrielle, un feu en train de mourir dans un bus abandonné (PF 43-45). Paul ranime le feu et le contemple longuement avec une sorte de fascination étrange (PF 45). Cette scène est significative, elle renvoie à la situation existentielle de Paul : déshumanisé par une société technique et industrielle, présente sous la forme de

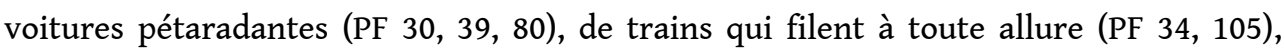
d'avions de combat qui traversent le ciel (PF 116), de centrale atomique (PF 84), de déchets industriels de toutes sortes (PF 43-45), Paul tente en rallumant le feu de la 
clairière de reconquérir symboliquement son intériorité, son humanité. Mais la tentative reste vaine, Paul n'éprouve aucun mouvement intérieur, il se contente de manger le gâteau, puis le silence de la clairière est envahi par le bruit tonitruant de moteurs, le cliquetis de chaînes, le grincement de roues sans que Paul ne parvienne à identifier la source et la nature de ces bruits (PF 46). La civilisation technique a repris ses droits, Paul s'éloigne de l'endroit et reprend son chemin. Avant de se coucher, Paul entreprend une traversée de la cave (PF 173-176), une sorte de descente symbolique en lui-même. Il n'y trouve que le fatras accumulé au cours des années, ustensiles de cuisines, matériel de bricolage, cartons, meubles, mobilier et outils de jardin. Significativement, la porte donnant accès au jardin est verrouillée (PF 176). Paul reste prisonnier de l'encombrement de lui-même. L'ultime promenade du 20 octobre se révèle en fait être une promenade labyrinthique, une quête vaine vers l'intérieur de soi-même, une impossibilité de voir clair en soi. Le vieux « labyrintheur ${ }^{18}$ a échoué à trouver son chemin de vérité dans le monde complexe, impénétrable, hostile qui est le sien, il reste alors ce vieil homme problématique pour lui-même, saisi par une angoisse muette, livré à une existence sans but.

La vieillesse de Paul se donne à voir exclusivement sous les aspects négatifs : déchéance physique, solitude, angoisse, vide existentiel. L'existence de cet homme s'est construite dans une société technique et industrielle, une société marchande qui pense les gens comme des choses, qui distingue les choses suivant leur rareté, et les hommes suivant leur puissance. Il n'y a plus chez Paul de vie intérieure, de retour sur soi, qui laisserait une place pour questionner le sens et cultiver l'espérance parce que son moi s'est réifié au contact d'une société dominée par le souffle froid de la rationalité technique, de la performance économique (le fameux miracle économique allemand) et du traitement instrumental des personnes. Paul a perdu «l'aptitude à participer de façon intéressée au monde dans lequel il intervient", il est privé de "l'ouverture qualitative à ce monde $»^{19}$.

\section{Chronique d'une fin annoncée : un avenir barré}

Est-il dès lors étonnant que cet homme soit voué à la mort? Les signes annonciateurs de sa mort traversent tout le récit. Ils sont présents dans la nature, dans son corps, dans les événements. Le récit se déroule le 20 octobre, une belle journée d'automne. Dans les premières pages du récit, Paul effleure un calendrier suspendu dans la cuisine, arrivé au 20 octobre, il laisse brusquement retomber son doigt et le narrateur ajoute ce commentaire " comme s'il avait dépassé une limite ${ }^{20}$, celle de sa vie. Paul constate les multiples signes du déclin de la nature : chute des feuilles (PF 7, 8, 14, 18, 26, 30 « lösten sich letzte Blätter », 37, 90), chute des fruits (PF 14, 57). Il se plaît à enregistrer la perte de puissance du vent qui se traduit par l'utilisation récurrente de verbes comme «abflauen» (PF 18, 30,76, 119) ou « ersterben» (PF 132). En observant les caprices du vent, il souligne cette sorte d'ultime expiration longue qui précède l'immobilité de la mort (PF 75-76, 119-120). Paul enregistre l'arrivée de l'hiver (PF 30 « das Stöhnen des nahenden Winters »). Ce qui retient son attention dans la nature laisse entendre que l'on s'achemine vers la fin de quelque chose, comme si Paul pressentait sa mort proche.

Le corps de Paul donne, lui aussi, tout au long du récit, de discrets signes annonciateurs d'un accident vasculaire cérébral. Paul enregistre les signaux d'alerte, furtifs au début du récit et massifs vers la fin, mais sans les reconnaître. À plusieurs reprises, il note un 
engourdissement de son bras (PF 115, 116, 188), un trouble de la sensibilité de ce bras (PF 61), une douleur fulgurante (PF 181-182), une altération de la vision (PF 184), une sensation de chaleur dans la tête ( $\mathrm{PF}$ 186). L'accident vasculaire cérébral se produit dans les dernières lignes du récit (PF 191-192), rien n'indique que Paul en réchappe. Avant l'accident Paul prend un bain, le narrateur prend soin de noter que le vieil homme sombre dans l'eau en un long mouvement continu jusqu'à ce que les flots se referment sur son corps qui s'efface, une sorte d'anticipation de la mort proche (PF 185).

La mort est enfin présente dans les différentes étapes de la promenade de Paul: le professeur Schneider évoque les obsèques de son beau frère devant Paul qui l'écoute d'une oreille distraite et indifférente (PF 74). Après sa visite, Paul entre dans le cimetière du village, au moment où il y découvre une tombe fraîchement ouverte, les cloches se mettent à sonner comme pour annoncer sa mort, le vieil homme s'effraie (PF 94). Enfin en sortant du cimetière Paul assiste à l'abattage d'un arbre qu'il voit tomber comme s'il était foudroyé (PF 96). Les signaux annonçant la mort sont trop nombreux dans le récit pour laisser un doute sur la mort de Paul. Celle-ci apparaît comme la conséquence logique et contraignante d'une existence improductive, inutile et vide qui avait perdu sa raison d'être. Le dernier mot, que prononce Paul, est «oui» (PF 192), comme s'il acquiesçait à sa mort.

\section{Conclusion}

La vieillesse de Paul n'est pas une vieillesse indigente du point de vue matériel, en tout cas rien dans le récit ne le laisse supposer, c'est un senior qui vit dans une société de la réussite économique et de l'abondance, et pourtant sa vieillesse est existentiellement d'une indigence abyssale. Tout indique dans le récit que Paul est privé de la maitrise de son passé comme de son présent, et donc de son humanité. Paul s'est construit au travers d'une histoire problématique par rapport à laquelle il ne semble pas être parvenu à faire le nécessaire travail de distanciation critique, ce passé tabou continue de provoquer en lui des attitudes réifiantes et déshumanisantes. Son existence s'est réalisée en interaction avec la société du miracle économique, une société vouée à la rationalité technique, à la performance, à la loi du plus fort. Ce que le lecteur perçoit de cette société apparaitt en creux à travers la vieillesse pitoyable de Paul. Le vieil homme n'a plus d'identité professionnelle, il n'est plus reconnu par la société, il n'exerce plus aucune action sur la société, il ne comprend plus la société dans laquelle il vit, il est réduit au rôle d'observateur d'une société dont il est exclu et qui lui est devenue étrangère. Privé de connaissance, de reconnaissance et d'action, il a perdu son humanité et sa raison de vivre, l'accident vasculaire cérébral apparaît alors comme la réponse somatique à ce vide existentiel. Quand le récit débute, la vie de Paul est déjà éteinte. Le récit ne fera que confirmer la réalité soulignée par le titre «la chute de Paul ». La mort de Paul confirme d'une certaine manière l'observation étonnante faite par Viktor Frankl dans les camps de concentration : « Face à l'absurde, les plus fragiles avaient développé une vie intérieure qui leur laissait une place pour garder l'espoir et questionner le sens, ils survivaient mieux que les plus robustes, qui étaient le plus dans l'action $^{21}$ ». Au cours de sa vie, Paul a manifestement participé à la reconstruction et au développement économique de la société allemande de l'après-guerre, il a été dans l'action, en revanche, pris dans l'engrenage de l'instrumentalisation des personnes, il s'est réifié, il a déserté son intériorité et a laissé s'effacer en lui l'espace où pouvait se 
poser la question du sens et se dessiner une utopie créatrice. Le rire de Paul à la fin de sa vie est celui d'un homme désespéré qui se sait au bord de l'abîme (PF 147).

La vieillesse de Paul, dérisoire parodie de la vie, résonne comme la terrible condamnation d'une société marchande sans solidarité, sans spiritualité, sans espérance. Elle souligne l'urgence absolue d'amener l'économie à faire sa révolution ${ }^{22}$ et la société à repenser la place et le rôle des seniors. Ce qui manque fondamentalement à Paul, c'est la possibilité d'être un acteur de sens dans une société qui ne se résume pas à la seule production de marchandises. L'exigence d'une vraie "retraite citoyenne $»^{23}$ met « tout le système en jeu et la revendication ne peut être que radicale : changer la vie $»^{24}$.

\section{NOTES}

1. Georges Minois, Histoire de la vieillesse en Occident : de l'Antiquité à la Renaissance, Fayard, Paris, 1987 ; Simone de Beauvoir, La Vieillesse. Essai, Gallimard, Paris, 1970. Pat Thane (Hg.), Das Alter. Eine Kulturgeschichte. Primus Verlag, Darmstadt, 2005.

2. Serge Guérin, L'Invention des seniors, Hachette Littératures, Paris, 2007.

3. À titre d'exemples on n'évoquera que quelques ouvrages récents : Martin Suter, Small World. Diogenes, Zürich, 1997 ; Björn Kern, Die Erlöser AG. C. H. Beck, München, 2007 ; Barbara Bronnen, Liebe bis in den Tod. Arche, Zürich, Hamburg, 2008; Arno Geiger, Der alte König im Exil. Hanser, München, 2011.

4. Arne Ross, Pauls Fall. Schöffling \& Co. Frankfurt a. M, 2006. Dans la suite, les références à ce récit seront indiquées par les initiales PF suivies de la page.

5. Cf. Andreas Müller, «Ein Monolith in der Literaturlandschaft. Selten hat sich ein Autor so konsequent gegen das Mitschwimmen im Strom des Zeitgeistes entschieden, noch seltener ist daraus ein so eindrucksvolles sprachliches Kunstwerk geworden. »Darmstädter Echo.

Voir aussi Oliver Seppelfricke, "Ein mutiger und äußerst kunstvoller Roman, der eine lange Tagesreise unternimmt. Lesenswert!»Saarländischer Rundfunk,http://www.schoeffling.de/ content/buecher/335.html, consulté le 16 novembre 2011.

OuChristoph Schröder, Frankfurter Rundschau du 15.3.2006: «Die konsequent durchgehaltene Ambivalenz und die Dezenz, in der Pauls Fall Fragen aufwirft, die an die Existenz gehen, machen diesen schmalen, stillen Roman zum Erlebnis. » http://www.lyrikwelt.de/rezensionen/paulsfallr.htm, consulté le 16 novembre 2011.

6. http://wm2006.deutschland.de/FR/Content/Le-pays-hote/Allemagne-en-bref/l-allemagnepole-economique.html, consulté le 10 décembre 2011.

7. http://www.lexpress.fr/actualites/1/monde/l-allemagne-a-la-meilleure-image-mondiale-lafrance-au-6e-rang_969682.html consulté le 10 décembre 2011.

8. Par son attitude Paul prive en quelque sorte son épouse de son humanité. Voir à propos de l'importance du regard Christian Bobin, L'Homme qui marche, Le temps qu'il fait, 1995, p. 12 : « La porte de l'humain, c'est le visage. Voir face à face, seul à seul, un à un. Dans les camps de concentration, les nazis interdisaient aux déportés de les regarder dans les yeux sous peine de mort immédiate. Celui dont je n'accueille plus le visage - et pour l'accueillir, il faut que je lave mon propre visage de toute matière de puissance - celui-là, je le vide de son humanité et je m'en vide moi-même. » 
9. La vie de Paul correspond parfaitement au diagnostic que pose Viktor Frankl sur le vide existentiel caractérisant notre époque : «Es ist nun gerade der Wille zum Sinn, der gegenwärtig weitgehend frustriert wird. In zunehmendem Masse bemächtigt sich des Menschen von heute ein Sinnlosigkeitsgefühl, das für gewöhnlich mit einem Gefühl der «inneren Leere » vergesellschaftet auftritt - es handelt sich um das von mir beschriebene und als solches bezeichnete " existentielle Vakuum ». Hauptsächlich manifestiert es sich in Form von Langweile und Gleichgültigkeit. Während in diesem Zusammenhang Langweile einen Verlust an Interesse bedeutet - Interesse an der Welt -, bedeutet Gleichgültikeit einen Mangel an Initiative - an der Initiative, in der Welt etwas zu verändern, etwa zu verbessern!» in: Viktor Frankl, Der unbewusste Gott. Psychotherapie und Religion, Deutscher Taschenbuch Verlag, München, 10. Auflage, 2010, p. 84.

10. Paul renverse le thé (PF 168), tache ses vêtements ( $P F$ 21), il ne parvient pas à enlever son manteau (PF 40).

11. Cf. Verena Krebs, "Horror ohne Schrecken. Arne Roß beschreibt die unerträgliche Langsamkeit des Seins.» Die Märkische. Voir aussi Carolina Schutti : «Meisterhaft versteht es Arne Roß, in der Langsamkeit und Ereignislosigkeit, dazu in einfacher, schlichter Sprache eine beinahe unerträgliche Spannung aufzubauen. Viel gibt es zu entdecken. Auch wenn der Protagonist ein alter Mann ist - PAULS FALL ist definitiv mehr als ein Roman über das Altern. » literaturkritik.de.http://www.schoeffling.de/content/buecher/335.html, consulté le 16 novembre 2011.

12. Voir Fragment 132 dans Les pensées de Blaise Pascal.

13. Avec une constance sans faille, Paul enregistre notamment les bruits de voitures (PF 30, 39, $46,53,80$ ) et de tondeuses (PF 37, 38, 40, 67).

14. À propos de la prière de Madame Thiele avant le repas, Paul affirme : «Wir sollten dieses Getue abschaffen [...], das bringt nichts. » (PF 166).

15. Le regard de Paul ne cesse de s'arrêter aux espaces clôturés (PF 43, 54, 62, 91, 93-94, 97, 114), à l'horizon barré (PF 52, 134, 105).

16. Cf. Bachelard, L'Eau et les rêves, essai sur l'imagination de la matière. Paris 1942.

17. La manière dont le regard de Paul traverse la maison, sans pouvoir s'y arrêter, et rejoint le jardin pour s'arrêter entre les troncs des sapins où se fait entendre une tronçonneuse, est très révélatrice: «... er spähte durch ein leeres, schattiges Zimmer in ein anderes, etwas helleres Zimmer und von dort durch ein zweites Fenster in den hinteren Garten, und im gleichen Augenblick sah er zwischen den Stämmen der Tannen etwas aufblitzen, er hörte das Gebrüll von Motoren, die zu kämpfen hatten, Räder schienen sich im Schlamm festzufahren, der Tannenwald war erfüllt von Lärm. » (PF 39).

18. Jacques Attali, Chemins de sagesse, traité du labyrinthe, Édition Fayard, Paris, 1996.

19. Axel Honneth, La réification. Petit traité de théorie critique, Paris, Gallimard, 2007, p. 44.

20. «Langsam fuhr er [Paul] mit dem linken Zeigefinger über die Zahlen des Zettels, ohne innezuhalten, dann, über dem Termin des zwanzigsten Oktober, liess er ihn plötzlich herabsacken, als sei er über eine Grenze hinausgeraten. » (PF 13).

21. Voir Viktor Frankl, « Un Psychiatre déporté témoigne », Éditions du Chalet, 1967.

22. Étienne Klein, Galilée et les Indiens, coll. « Café Voltaire », Flammarion, Paris, 2008.

23. Serge Guérin, La Société des seniors. Essai. Michalon, Paris, 2009, p. 100.

24. Simone de Beauvoir, La Vieillesse. Essai. Gallimard, Paris, 1970, p. 570. 


\section{RÉSUMÉS}

La Chute de Paul d'Arne Ross (2006) retrace la dernière journée de vie d'un vieil homme vivant dans la société de l'abondance de l'Allemagne du xxie siècle. Ce roman se distingue de la production récente sur le thème de la vieillesse par l'attention portée à la dimension existentielle de cet âge. L'analyse tend à montrer que l'indigence existentielle de cette vieillesse trouve sa source dans un passé non assumé, un présent vide de sens et d'intériorité et un avenir sans perspective. Privé de la compréhension de cette société, de la reconnaissance de soi par cette société et de la possibilité d'agir sur cette société, le personnage principal s'est réifié, il a perdu son humanité et sa raison de vivre. L'accident vasculaire cérébral apparaît alors comme la réponse somatique à ce vide existentiel. Le vieillesse de Paul, dérisoire parodie de la vie, résonne comme la terrible condamnation d'une société marchande sans solidarité, sans spiritualité, sans espérance. Elle souligne l'urgence absolue d'amener l'économie à faire sa révolution et la société à repenser la place et le rôle des seniors.

Pauls Fall (Paul's Case) by Arne Ross (2006) recalls the last day of an old man who lives in the affluent society of $21^{\text {st }}$ century Germany. This novel distinguishes itself from recent books on old age in that it addresses the existential challenges of that stage of life. Our analysis suggests that the existential indigence of the aged finds its origin in an unaccepted past, a meaningless present, devoid of interiority, and a perspectiveless future. The main character has been reified as a result of his inability to comprehend, be valued by and exert an influence on the society he lives in. He has lost his humanity and his reason to survive. The cerebrovascular accident then appears as a somatic response to this existential void. Paul's old age is a derisory parody of life, which echoes the terrible condemnation of a mercantile society that has lost the sense of solidarity, spirituality and hope. It underlines the urgent need for radical economic changes and a new approach to the place and role of the elderly in contemporary society.

Der Roman Pauls Fall von Arne Ross schildert den letzten Lebenstag eines alten Mannes, der in der deutschen Überflussgesellschaft des 21. Jahrhunderts lebt. Der Roman unterscheidet sich von der sich mit der Altersproblematik beschäftigenden literarischen Produktion der letzten Jahre dadurch, dass darin die existentielle Dimension des Alters besondere Berücktsichtigung erfährt. Die Analyse zielt darauf ab zu zeigen, dass die existentielle Not der Hauptfigur ihren Grund in der unbewältigten Vergangenheit sowie in einer als sinnlos und oberflächlich empfundenen Gegenwart und in einer Zukunft ohne Perspektive findet. Da dem Protagonisten das Verständnis der Gesellschaft, die Anerkennung seiner selbst durch diese Gesellschaft und die Möglichkeit, auf diese Gesellschaft einzuwirken, abhanden gekommen ist, hat er sich verdinglicht ; er hat seine Humanität und den Mut zum Leben verloren. Der Hirnschlag erscheint alsdann als die somatische Antwort auf die existentielle Sinnleere. Pauls Altersleben, eine lächerliche Parodie des Lebens, wirkt wie ein vernichtendes Urteil über eine Überflussgesellschaft ohne Solidarität, ohne Geistigkeit, ohne Hoffnung. Es deutet auf die absolute Notwendigkeit einer Revolution in der Wirtschaft und einer neuen Definition der Rolle der alten Menschen in der Gesellschaft hin.

\section{INDEX}

Mots-clés : Allemagne, intériorité, roman, vide existentiel, vieillesse

oeuvrecitee Pauls Fall 
AUTEUR

BERNARD BACH

Université Charles-de-Gaulle - Lille 3 